\title{
ON THE STRONG ARNOL'D HYPOTHESIS AND THE CONNECTIVITY OF GRAPHS*
}

\author{
HEIN VAN DER HOLST ${ }^{\dagger}$
}

\begin{abstract}
In the definition of the graph parameters $\mu(G)$ and $\nu(G)$, introduced by Colin de Verdière, and in the definition of the graph parameter $\xi(G)$, introduced by Barioli, Fallat, and Hogben, a transversality condition is used, called the Strong Arnol'd Hypothesis. In this paper, we define the Strong Arnol'd Hypothesis for linear subspaces $L \subseteq \mathbb{R}^{n}$ with respect to a graph $G=(V, E)$, with $V=\{1,2, \ldots, n\}$. We give a necessary and sufficient condition for a linear subspace $L \subseteq \mathbb{R}^{n}$ with $\operatorname{dim} L \leq 2$ to satisfy the Strong Arnol'd Hypothesis with respect to a graph $G$, and we obtain a sufficient condition for a linear subspace $L \subseteq \mathbb{R}^{n}$ with $\operatorname{dim} L=3$ to satisfy the Strong Arnol'd Hypothesis with respect to a graph $G$. We apply these results to show that if $G=(V, E)$ with $V=\{1,2, \ldots, n\}$ is a path, 2-connected outerplanar, or 3-connected planar, then each real symmetric $n \times n$ matrix $M=\left[m_{i, j}\right]$ with $m_{i, j}<0$ if $i j \in E$ and $m_{i, j}=0$ if $i \neq j$ and $i j \notin E$ (and no restriction on the diagonal), having exactly one negative eigenvalue, satisfies the Strong Arnol'd Hypothesis.
\end{abstract}

Key words. Symmetric matrices, Nullity, Graphs, Transversality, Planar, Outerplanar, Graph minor.

AMS subject classifications. 05C50, 15A18.

1. Introduction. In the definition of the graph parameters $\mu(G)$ and $\nu(G)$, introduced by Colin de Verdière in respectively [2,3] and [4], and in the definition of the graph parameter $\xi(G)$, introduced by Barioli, Fallat, and Hogben in [1], a transversality condition is used, called the Strong Arnol'd Hypothesis. The addition of this Strong Arnol'd Hypothesis allows to show the minor-monotonicity of these graph parameters. For example, $\mu\left(G^{\prime}\right) \leq \mu(G)$ if $G^{\prime}$ is a minor of $G$; we refer to Diestel [5] for the notions used in graph theory. It is this minor-monotonicity that makes these graph parameters so useful.

Let us first recall the definition of the Strong Arnol'd Hypothesis. For a graph $G=(V, E)$ with vertex set $V=\{1,2, \ldots, n\}$, denote by $\mathcal{S}(G)$ the set of all real symmetric $n \times n$ matrices $M=\left[m_{i, j}\right]$ with

$$
m_{i, j} \neq 0, i \neq j \Leftrightarrow i j \in E \text {. }
$$

The tangent space, $T_{M} \mathcal{S}(G)$, of $\mathcal{S}(G)$ at $M$ is the space of all real symmetric $n \times n$

\footnotetext{
*Received by the editors November 12, 2007. Accepted for publication on July 31, 2010. Handling Editor: Richard A. Brualdi.

†School of Mathematics, Georgia Institute of Technology, Atlanta, GA 30332-0160, USA (holst@math.gatech.edu). On leave from Eindhoven University of Technology.
} 
matrices $A=\left[a_{i, j}\right]$ with $a_{i, j}=0$ if $i \neq j$ and $i$ and $j$ are nonadjacent. Denote by $\mathcal{R}_{n, k}$ the manifold of all real symmetric $n \times n$ matrices of nullity $k$. The tangent space, $T_{M} \mathcal{R}_{n, k}$, of $\mathcal{R}_{n, k}$ at $M$ is the space of all real symmetric $n \times n$ matrices $B=\left[b_{i, j}\right]$ such that $x^{T} B x=0$ for all $x \in \operatorname{ker}(M)$. Here, $\operatorname{ker}(M)$ denotes the null space of $M$. A matrix $M \in \mathcal{S}(G)$ satisfies the Strong Arnol'd Hypothesis if the sum of $T_{M} \mathcal{S}(G)$ and $T_{A} \mathcal{R}_{n, k}$ equals the space of all real symmetric $n \times n$ matrices. So, a matrix $M \in \mathcal{S}(G)$ satisfies the Strong Arnol'd Hypothesis if and only if for each real symmetric $n \times n$ matrix $B$, there is a real symmetric matrix $A=\left[a_{i, j}\right]$ with $a_{i, j}=0$ if $i \neq j$ and $i$ and $j$ nonadjacent, such that $x^{T} B x=x^{T} A x$ for each $x \in \operatorname{ker}(M)$.

Although stated above as a condition on the matrix $M$, it can be viewed as a condition on $\operatorname{ker}(M)$. In this paper, we extend the definition of the Strong Arnol'd Hypothesis to linear subspaces $L \subseteq \mathbb{R}^{n}$ with respect to a graph $G=(V, E)$, where $V=\{1,2, \ldots, n\}$. We give a necessary and sufficient condition for a linear subspace $L \subseteq \mathbb{R}^{n}$ with $\operatorname{dim} L \leq 2$ to satisfy the Strong Arnol'd Hypothesis with respect to a graph $G$, and we obtain a sufficient condition for a linear subspace $L \subseteq \mathbb{R}^{n}$ with $\operatorname{dim} L=3$ to satisfy the Strong Arnol'd Hypothesis with respect to a graph $G$.

For a graph $G=(V, E)$, let $\mathcal{O}(G)$ be the set of all $M=\left[m_{i, j}\right] \in \mathcal{S}(G)$ such that $m_{i, j}<0$ for each adjacent pair of vertices $i$ and $j$. Notice that for a matrix $M \in \mathcal{O}(G)$ with exactly one negative eigenvalue, the tangent space of $\mathcal{O}(G)$ at $M$ is the same as the tangent space of $\mathcal{S}(G)$ at $M$. The parameter $\mu(G)$ is defined as the largest nullity of any $M=\left[m_{i, j}\right] \in \mathcal{O}(G)$ such that $M$ has exactly one negative eigenvalue and satisfies the Strong Arnol'd Hypothesis. This graph parameter characterizes outerplanar graphs as those graphs $G$ for which $\mu(G) \leq 2$, and planar graphs as those graphs $G$ for which $\mu(G) \leq 3$; see van der Holst, Lovász, and Schrijver [9] for an introduction to this graph parameter. We show that in certain cases each $M \in \mathcal{O}(G)$ with exactly one negative eigenvalue (automatically) satisfies the Strong Arnol'd Hypothesis. More precisely, if $G$ is a path, 2-connected outerplanar, or 3connected planar, then each $M \in \mathcal{O}(G)$ with exactly one negative eigenvalue satisfies the Strong Arnol'd Hypothesis.

2. The Strong Arnol'd Property for linear subspaces. A representation of linearly independent vectors $x_{1}, x_{2}, \ldots, x_{r} \in \mathbb{R}^{n}$ is a function $\phi:\{1,2, \ldots, n\} \rightarrow \mathbb{R}^{r}$ such that

$$
\left[\begin{array}{llll}
\phi(1) & \phi(2) & \ldots & \phi(n)
\end{array}\right]=\left[\begin{array}{c}
x_{1}^{T} \\
x_{2}^{T} \\
\vdots \\
x_{r}^{T}
\end{array}\right] .
$$

A representation of a linear subspace $L$ of $\mathbb{R}^{n}$ is a representation of some basis of $L$. 
Let $\phi:\{1,2, \ldots, n\} \rightarrow \mathbb{R}^{r}$ be a representation of a basis $x_{1}, x_{2}, \ldots, x_{r}$ of a linear subspace $L$ of $\mathbb{R}^{n}$, and let $G=(V, E)$ be a graph with vertex set $V=\{1,2, \ldots, n\}$. If $A$ is a nonsingular $r \times r$ matrix and the linear span of the symmetric $r \times r$ matrices $\phi(i) \phi(i)^{T}, i \in V$, and $\phi(i) \phi(j)^{T}+\phi(j) \phi(i)^{T}, i j \in E$, is equal to the space of all symmetric $r \times r$ matrices, then the same holds for the linear span of $A \phi(i) \phi(i)^{T} A^{T}$, $i \in V$, and $A \phi(i) \phi(j)^{T} A^{T}+A \phi(j) \phi(i)^{T} A^{T}, i j \in E$. This suggests to define the following property for linear subspaces of $\mathbb{R}^{n}$.

An $r$-dimensional linear subspace $L$ of $\mathbb{R}^{n}$ satisfies the Strong Arnol'd Hypothesis with respect to $G$ if for any representation $\phi:\{1,2, \ldots, n\} \rightarrow \mathbb{R}^{r}$ of a basis of $L$, the linear span of all matrices of the form $\phi(i) \phi(i)^{T}, i \in V$, and $\phi(i) \phi(j)^{T}+\phi(j) \phi(i)^{T}$, $i j \in E$, is equal to the space of all symmetric $r \times r$ matrices. Equivalently, an $r$ dimensional linear subspace $L$ of $\mathbb{R}^{n}$ satisfies the Strong Arnol'd Hypothesis if the $r \times r$ all-zero matrix is the only symmetric $r \times r$ matrix $N$ such that $\phi(i)^{T} N \phi(j)=0$, $i j \in E$, and $\phi(i)^{T} N \phi(i)=0, i \in V$. If it is clear what graph $G$ we are dealing with, we only write that $L$ satisfies the Strong Arnol'd Hypothesis, omitting the part with respect to $G$.

The next lemma shows why we call this property the Strong Arnol'd Hypothesis.

Lemma 2.1. Let $G=(V, E)$ be a graph with vertex set $V=\{1,2, \ldots, n\} . A$ matrix $M \in \mathcal{S}(G)$ has the Strong Arnol'd Hypothesis if and only if $\operatorname{ker}(M)$ has the Strong Arnol'd Hypothesis.

Proof. Choose a basis $x_{1}, x_{2}, \ldots, x_{r}$ of $\operatorname{ker}(M)$, and let $\phi$ be a representation of $x_{1}, x_{2}, \ldots, x_{r}$.

$M$ satisfies the Strong Arnol'd Hypothesis if and only if for every symmetric $n \times n$ matrices $A$, there is a symmetric $n \times n$ matrix $B=\left[b_{i, j}\right]$ with $b_{i, j}=0$ if $i \neq j$ and $i$ and $j$ are nonadjacent, such that for all $x \in \operatorname{ker}(M), x^{T} A x=x^{T} B x$. Hence, $M$ has the Strong Arnol'd Hypothesis if and only if for every symmetric $r \times r$ matrices $C$, there is a symmetric $n \times n$ matrix $B=\left[b_{i, j}\right]$ with $b_{i, j}=0$ if $i \neq j$ and $i$ and $j$ are nonadjacent, such that

$$
C=\left[\begin{array}{lll}
x_{1} & \ldots & x_{r}
\end{array}\right]^{T} B\left[\begin{array}{lll}
x_{1} & \ldots & x_{r}
\end{array}\right]
$$

This is equivalent to: $M$ has the Strong Arnol'd Hypothesis if and only if the linear span of all matrices of the form $\phi(i) \phi(i)^{T}, i \in V$, and $\phi(i) \phi(j)^{T}+\phi(j) \phi(i)^{T}, i j \in E$, is equal to the space of all symmetric $r \times r$ matrices.

Let $G=(V, E)$ be a graph. For $S \subseteq V$, we denote by $N(S)$ the set of all vertices in $V \backslash S$ adjacent to a vertex in $S$, and we denote by $G[S]$ the subgraph induced by $S$. For $x \in \mathbb{R}^{n}$, we denote $\operatorname{supp}(x)=\left\{i \mid x_{i} \neq 0\right\}$. Two subsets of the vertex set or two subgraphs of a graph touch if they have common vertex or are adjacent. If two 
subsets of the vertex set or two subgraphs of a graph do not touch, then we say that they are separated.

LEMma 2.2. Let $L$ be a linear space of $\mathbb{R}^{n}$ of dimension $r$ and let $\phi: V \rightarrow \mathbb{R}^{r}$ be a representation of the basis $x_{1}, x_{2}, \ldots, x_{r}$ of $L$. Then there is a symmetric $r \times r$ matrix $N=\left[n_{i, j}\right]$ with $n_{1,2}=n_{2,1}=1$ and $n_{i, j}=0$ elsewhere, such that $\phi(i)^{T} N \phi(i)=0$ for all $i \in V$ and $\phi(i)^{T} N \phi(j)=0$ for all $i j \in E$ if and only if $\operatorname{supp}\left(x_{1}\right)$ and $\operatorname{supp}\left(x_{2}\right)$ are separated.

Proof. It is easily checked that $\phi(i)^{T} N \phi(i)=0$ for all $i \in V$ and $\phi(i)^{T} N \phi(j)=0$ for all $i j \in E$ if $\operatorname{supp}\left(x_{1}\right)$ and $\operatorname{supp}\left(x_{2}\right)$ are separated.

Conversely, from $\phi(i)^{T} N \phi(i)=0, i \in V$, it follows that $\operatorname{supp}\left(x_{1}\right)$ and $\operatorname{supp}\left(x_{2}\right)$ have no common vertex, and from $\phi(i)^{T} N \phi(j)=0, i j \in E$, it follows that $\operatorname{supp}\left(x_{1}\right)$ and $\operatorname{supp}\left(x_{2}\right)$ are not adjacent. Hence, $\operatorname{supp}\left(x_{1}\right)$ and $\operatorname{supp}\left(x_{2}\right)$ are separated.

If a linear subspace $L \subseteq \mathbb{R}^{n}$ has $\operatorname{dim} L \leq 2$, then the following theorem gives a sufficient and necessary condition for $L$ to satisfy the Strong Arnol'd Hypothesis with respect to $G$.

TheOREM 2.3. Let $G=(V, E)$ be a graph with vertex set $V=\{1,2, \ldots, n\}$ and let $k \leq 2$. A $k$-dimensional linear subspace $L$ of $\mathbb{R}^{n}$ does not satisfy the Strong Arnol'd Hypothesis if and only if there are nonzero vectors $x_{1}, x_{2} \in L$ such that $\operatorname{supp}\left(x_{1}\right)$ and $\operatorname{supp}\left(x_{2}\right)$ are separated.

Proof. $k=1$. This is easy as every 1 -dimensional linear subspace $L$ satisfies the Strong Arnol'd Hypothesis, and there are no two nonzero vectors $x_{1}, x_{2} \in L$ such that $\operatorname{supp}\left(x_{1}\right)$ and $\operatorname{supp}\left(x_{2}\right)$ are separated.

$k=2$. If there are nonzero vectors $x_{1}, x_{2} \in L$ for which $\operatorname{supp}\left(x_{1}\right)$ and $\operatorname{supp}\left(x_{2}\right)$ are separated, then $L$ does not satisfy the Strong Arnol'd Hypothesis, by Lemma 2.2.

Conversely, suppose that $L$ does not satisfy the Strong Arnol'd Hypothesis. Since $L$ has dimension 2, we can find two vertices $u$ and $v$ and a basis $x, z$ of $L$ with $x_{u}=1, z_{u}=0$ and $x_{v}=0, z_{v}=1$. Let $\phi: V \rightarrow \mathbb{R}^{2}$ be a representation of $x, z$. As $L$ does not satisfy the Strong Arnol'd Hypothesis, there is a nonzero symmetric $2 \times 2$ matrix $N=\left[n_{i, j}\right]$ such that $\phi(i)^{T} N \phi(i)=0$ for all $i \in V$ and $\phi(i)^{T} N \phi(j)=0$ for all $i j \in E$. In particular, since $\phi(u)=[1,0]^{T}$ and $\phi(v)=[0,1]^{T}, n_{1,1}=n_{2,2}=0$. Hence, by Lemma $2.2, \operatorname{supp}\left(x_{1}\right)$ and $\operatorname{supp}\left(x_{2}\right)$ are separated.

Theorem 2.3 need not hold when $\operatorname{dim} L=3$, as the following example shows. Let $G=(V, E)$ be the graph with $V=\{1,2, \ldots, 5\}$ and $E=\emptyset$, and let $L$ be the linear 
subspace of $\mathbb{R}^{5}$ spanned by the vectors

$$
\left[\begin{array}{l}
1 \\
0 \\
0 \\
1 \\
1
\end{array}\right],\left[\begin{array}{l}
0 \\
1 \\
0 \\
1 \\
2
\end{array}\right],\left[\begin{array}{l}
0 \\
0 \\
1 \\
1 \\
3
\end{array}\right] .
$$

Every two nonzero vectors $x_{1}, x_{2} \in L$ touch, but $L$ does not satisfy the Strong Arnol'd Hypothesis, as can be easily verified.

If a linear subspace $L \subseteq \mathbb{R}^{n}$ has $\operatorname{dim} L=3$, then the following theorem gives a sufficient condition for $L$ to satisfy the Strong Arnol'd Hypothesis.

TheOREM 2.4. Let $G=(V, E)$ be a graph with vertex set $V=\{1,2, \ldots, n\}$, and let $L$ be a linear subspace of $\mathbb{R}^{n}$ with $\operatorname{dim} L=3$. Let $\phi: V \rightarrow \mathbb{R}^{3}$ be a representation of $L$. If there are adjacent vertices $u$ and $v$ in $G$ such that $\phi(u)$ and $\phi(v)$ are linearly independent, and there are no nonzero vectors $x_{1}, x_{2} \in L$ such that $\operatorname{supp}\left(x_{1}\right)$ and $\operatorname{supp}\left(x_{2}\right)$ are separated, then L satisfies the Strong Arnol'd Hypothesis.

Proof. For the sake of contradiction, assume that $L$ does not satisfy the Strong Arnol'd Hypothesis. Then there is a nonzero symmetric $3 \times 3$ matrix $N=\left[n_{i, j}\right]$ such that $\phi(i)^{T} N \phi(i)=0$ for all $i \in V$ and $\phi(i)^{T} N \phi(j)=0$ for all $i j \in E$. There exists a nonsingular matrix $A$ such that $A^{T} N A$ is a diagonal matrix in which each of the diagonal entries belongs to $\{-1,0,1\}$. Thus, by multiplying $\phi$ with $A$ we may assume that $N$ is a diagonal matrix and that its diagonal entries belongs to $\{-1,0,1\}$. We will now show that each of the elements in $\{-1,0,1\}$ occurs as a diagonal entry.

Suppose that 0 occurs twice as a diagonal entry; without loss of generality, we may assume that $n_{2,2}=n_{3,3}=0$. Since the dimension of $L$ is three, there exists a vertex $v$ for which the first coordinate of $\phi(v)$ is nonzero. Then $\phi(v)^{T} N \phi(v) \neq 0$, contradicting that $\phi(i)^{T} N \phi(i)=0$ for all $i \in V$.

Suppose that 1 occurs twice as a diagonal entry; without loss of generality, we may assume that $n_{2,2}=n_{3,3}=1$. Since $\phi(u)$ and $\phi(v)$ are linearly independent, there exists a linear combination $z=a \phi(u)+b \phi(v)$ for which the first coordinate equals 0 . Then $0 \neq z^{T} N z=a^{2} \phi(u)^{T} N \phi(u)+2 a b \phi(u)^{T} N \phi(v)+b^{2} \phi(v)^{T} N \phi(v)$. Since $\phi(u)^{T} N \phi(u)=0, \phi(v)^{T} N \phi(v)=0$, and $\phi(u)^{T} N \phi(v)=0$, we obtain a contradiction. The case where -1 occurs twice is analogous.

Hence, each of the elements in $\{-1,0,1\}$ occurs as a diagonal entry; we may assume that

$$
N=\left[\begin{array}{ccc}
1 & 0 & 0 \\
0 & -1 & 0 \\
0 & 0 & 0
\end{array}\right]
$$


We now define $\psi: V \rightarrow \mathbb{R}^{3}$ by $\psi(i)=B \phi(i)$ for $i \in V$, where

$$
B=\left[\begin{array}{ccc}
1 & -1 & 0 \\
\frac{1}{2} & \frac{1}{2} & 0 \\
0 & 0 & 1
\end{array}\right] .
$$

Then $\psi$ is a representation of $L$ such that if

$$
Q=\left[\begin{array}{lll}
0 & 1 & 0 \\
1 & 0 & 0 \\
0 & 0 & 0
\end{array}\right],
$$

then $\psi(i)^{T} Q \psi(i)=0$ for all $i \in V$ and $\psi(i)^{T} Q \psi(j)=0$ for all $i j \in E$. By Lemma 2.2, there are nonzero vectors $x_{1}, x_{2} \in L \operatorname{such}$ that $\operatorname{supp}\left(x_{1}\right)$ and $\operatorname{supp}\left(x_{2}\right)$ are separated, contradicting the assumption. Hence, $L$ satisfies the Strong Arnol'd Hypothesis.

Lemma 2.5. Let $G=(V, E)$ be a graph. Let $\phi: V \rightarrow \mathbb{R}^{3}$ be a representation of a linear subspace $L$ of $\mathbb{R}^{n}$ with $\operatorname{dim} L=3$. If there are nonzero vectors $x_{1}, x_{2} \in L$ for which there are touching components $C_{1}$ and $C_{2}$ of $G\left[\operatorname{supp}\left(x_{1}\right)\right]$ and $G\left[\operatorname{supp}\left(x_{2}\right)\right]$, respectively, with $C_{1} \neq C_{2}$, then there are adjacent vertices $u$ and $v$ such that $\phi(u)$ and $\phi(v)$ are independent.

Proof. The vectors $x_{1}, x_{2}$ are clearly linearly independent. Let $x_{3}$ be a vector in $L$ such that $x_{1}, x_{2}, x_{3}$ form a basis of $L$, and let $\psi: V \rightarrow \mathbb{R}^{3}$ be a representation of $x_{1}, x_{2}, x_{3}$. If for adjacent vertices $u$ and $v, \psi(u)$ and $\psi(v)$ are linearly independent, then also $\phi(u)$ and $\phi(v)$ are linearly independent.

If $C_{1}$ and $C_{2}$ have no vertex in common, then they must be joined by an edge $u v$. As a consequence, $\psi(u)$ and $\psi(v)$ are linear independent, and so $\phi(u)$ and $\phi(v)$ are linearly independent.

We may therefore assume that $C_{1}$ and $C_{2}$ have a vertex $c$ in common. Since $C_{1} \neq C_{2}, V\left(C_{1}\right) \Delta V\left(C_{2}\right) \neq \emptyset$; choose a vertex $d$ from $V\left(C_{1}\right) \Delta V\left(C_{2}\right)$. By symmetry, we may assume that $d \in V\left(C_{1}\right)$ and $d \notin V\left(C_{2}\right)$. Since $C_{1}$ and $C_{2}$ are connected, there is a path in $C_{1}$ connecting $c$ and $d$. On this path there is an edge $u v$ such that $u \in V\left(C_{1}\right), u \notin V\left(C_{2}\right)$ and $v \in V\left(C_{1}\right), v \in V\left(C_{2}\right)$. Then $\psi(u)$ and $\psi(v)$ are linear independent. Hence, $\phi(u)$ and $\phi(v)$ are linearly independent.

Using Theorem 2.4 and Lemma 2.5, we obtain:

TheOREM 2.6. Let $G=(V, E)$ be a graph. Let $\phi: V \rightarrow \mathbb{R}^{3}$ be a representation of a linear subspace $L$ of $\mathbb{R}^{n}$ with $\operatorname{dim} L=3$. If there are nonzero vectors $x_{1}, x_{2} \in L$ for which there are touching components $C_{1}$ and $C_{2}$ of $G\left[\operatorname{supp}\left(x_{1}\right)\right]$ and $G\left[\operatorname{supp}\left(x_{2}\right)\right]$, respectively, with $C_{1} \neq C_{2}$, then L satisfies the Strong Arnol'd Hypothesis.

Lemma 2.7. Let $G=(V, E)$ be a graph with vertex set $V=\{1,2, \ldots, n\}$, and let $L$ be a linear subspace of $\mathbb{R}^{n}$ with $\operatorname{dim} L \leq 3$, which has a nonzero vector $x$ such that 
$G[\operatorname{supp}(x)]$ is connected. If $L$ does not satisfy the Strong Arnol'd Hypothesis, then there exists a nonzero vector $y \in L$ such that $\operatorname{supp}(x)$ and $\operatorname{supp}(y)$ are separated.

Proof. If each nonzero vector $y \in L$ satisfies $\operatorname{supp}(y)=\operatorname{supp}(x)$, then $L$ is 1dimensional; each 1-dimensional linear subspace $L$ of $\mathbb{R}^{n}$ satisfies the Strong Arnol'd Hypothesis.

Thus, there exists a nonzero vector $y \in L$ such that $\operatorname{supp}(y) \neq \operatorname{supp}(x)$. We may assume that $\operatorname{supp}(x)$ and $\operatorname{supp}(y)$ touch, for otherwise $\operatorname{supp}(x)$ and $\operatorname{supp}(y)$ are separated. Hence, there is a component $C$ of $G[\operatorname{supp}(y)]$ such that $G[\operatorname{supp}(x)]$ and $C$ touch. If $C \neq G[\operatorname{supp}(x)]$, then $L$ would satisfy the Strong Arnol'd Hypothesis by Theorem 2.6. This contradiction shows that $C=G[\operatorname{supp}(x)]$. Now choose a vertex $v \in \operatorname{supp}(x)$. There exists a scalar $\alpha$ such that $z=\alpha x+y$ satisfies $z_{v}=0$. If there is a vertex $w \in \operatorname{supp}(x)$ such that $z_{w} \neq 0$, then there is a component $D$ of $G[\operatorname{supp}(z)]$ such that $D$ and $G[\operatorname{supp}(x)]$ touch and $D \neq G[\operatorname{supp}(x)]$. By Theorem 2.6, $L$ would satisfy the Strong Arnol'd Hypothesis. This contradiction shows that $z_{u}=0$ for all $u \in G[\operatorname{supp}(x)]$. Then $\operatorname{supp}(x)$ and $\operatorname{supp}(z)$ are separated.

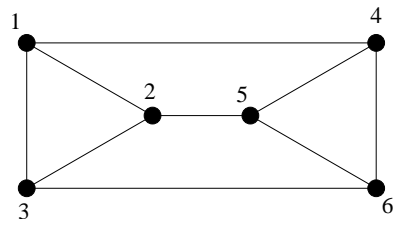

FIG. 2.1. Complement of $C_{6}$.

In Theorem 2.4, the restriction $k \leq 3$ cannot be removed. For $k=4$, there is the following example. Let $G=(V, E)$ be the complement of the 6-cycle $C_{6}$, which is the graph with $V=\{1,2, \ldots, 6\}$ obtained from taking two disjoint triangles and connecting each vertex of one triangle to a vertex of the other triangle by an edge in a one-to-one way; see Figure 2.1. Let $L$ be generated by the columns of the matrix

$$
A=\left[\begin{array}{cccc}
1 & 0 & 0 & 0 \\
-1 & 1 & 0 & 0 \\
0 & -1 & 0 & 0 \\
0 & 0 & 1 & 0 \\
0 & 0 & -1 & 1 \\
0 & 0 & 0 & -1
\end{array}\right]
$$

and, for $i \in V$, let $\phi(i)$ be the $i$ th column of $A^{T}$. Then for every vector $x \in L, \operatorname{supp}(x)$ induces a connected subgraph of $G$, and hence, for every two vectors $x_{1}, x_{2} \in L$, $\operatorname{supp}\left(x_{1}\right)$ and $\operatorname{supp}\left(x_{2}\right)$ touch. But $L$ does not satisfy the Strong Arnol'd Hypothesis, 
as $\phi(i)^{T} Q \phi(i)=0$ for $i \in V$ and $\phi(i)^{T} Q \phi(j)=0$ for $i j \in E$ if

$$
Q=\left[\begin{array}{llll}
0 & 0 & 0 & 1 \\
0 & 0 & 1 & 0 \\
0 & 1 & 0 & 0 \\
1 & 0 & 0 & 0
\end{array}\right]
$$

However, this is essentially the only type of matrix that can occur as we will see in the next result.

TheOrem 2.8. Let $G=(V, E)$ be a graph with vertex set $V=\{1, \ldots, n\}$, and let $L$ be a linear subspace of $\mathbb{R}^{n}$ with $\operatorname{dim} L=4$. Let $\phi: V \rightarrow \mathbb{R}^{4}$ be a representation of L. Suppose $L$ has the following properties:

1. L does not satisfy the Strong Arnol'd Hypothesis,

2. there are adjacent vertices $u$ and $w$ in $G$ such that $\phi(u)$ and $\phi(w)$ are linearly independent, and

3. there are no nonzero vectors $x_{1}, x_{2} \in L$ such that $\operatorname{supp}\left(x_{1}\right)$ and $\operatorname{supp}\left(x_{2}\right)$ are separated.

Then there is a representation $\psi: V \rightarrow \mathbb{R}^{4}$ of $L$ such that if

$$
Q=\left[\begin{array}{llll}
0 & 0 & 0 & 1 \\
0 & 0 & 1 & 0 \\
0 & 1 & 0 & 0 \\
1 & 0 & 0 & 0
\end{array}\right]
$$

then $\psi(i)^{T} Q \psi(i)=0$ for all $i \in V$ and $\psi(i)^{T} Q \psi(j)=0$ for all $i j \in E$.

Proof. Since $L$ does not satisfy the Strong Arnol'd Hypothesis, there is a nonzero symmetric $4 \times 4$ matrix $N=\left[n_{i, j}\right]$ such that $\phi(v)^{T} N \phi(v)=0$ for each $v \in V$ and $\phi(v)^{T} N \phi(w)=0$ for each $v w \in E$. By multiplying $\phi$ with a nonsingular $4 \times 4$ matrix $A$, we may assume that $N$ is a diagonal matrix and that each of its diagonal entries belongs to $\{-1,0,1\}$.

Suppose first that three of the diagonal entries are equal to zero; without loss of generality, we may assume that $n_{1,1}=n_{2,2}=n_{3,3}=0$. Since $\operatorname{dim} L=4$, there exists a vertex $v$ such that the last coordinate of $\phi(v)$ is nonzero. Then $\phi(v)^{T} N \phi(v) \neq 0$. This contradiction shows that at most two of the diagonal entries are equal to zero.

Suppose next that two of the diagonal entries are equal to zero; without loss of generality, we may assume that $n_{1,1}=n_{2,2}=0$. If $n_{3,3}=n_{4,4}$, then $\phi(v)^{T} N \phi(v) \neq 0$. 
Hence, $n_{3,3}=-n_{4,4}$; we may assume that $n_{3,3}=1$. Taking

$$
A=\left[\begin{array}{cccc}
1 & 0 & 0 & 0 \\
0 & 1 & 0 & 0 \\
0 & 0 & 1 & \frac{1}{2} \\
0 & 0 & -1 & \frac{1}{2}
\end{array}\right],
$$

we obtain

$$
A^{T} N A=\left[\begin{array}{llll}
0 & 0 & 0 & 0 \\
0 & 0 & 0 & 0 \\
0 & 0 & 0 & 1 \\
0 & 0 & 1 & 0
\end{array}\right] .
$$

Let $\psi: V \rightarrow \mathbb{R}^{4}$ be defined by $\psi(i)=A^{-1} \phi(i)$ for $i=1,2, \ldots, n$. Then, by Lemma 2.2 , there exist vectors $x_{1}, x_{2} \in L$ such that $\operatorname{supp}\left(x_{1}\right)$ and $\operatorname{supp}\left(x_{2}\right)$ are separated, contradicting the assumption.

Suppose next that exactly one of the diagonal entries is equal to zero; without loss of generality, we may assume that $n_{4,4}=0$. Each of the other diagonal entries is -1 or 1 . Let $z_{1}, z_{2}, z_{3}, z_{4}$ be the basis corresponding to $\phi$ and let $\psi$ be the representation corresponding to $z_{1}, z_{2}, z_{3}$. If $R=\left[r_{i, j}\right]$ is the diagonal matrix defined by $r_{j, j}=n_{j, j}$ for $j=1,2,3$, then $\psi(v)^{T} R \psi(v)=0$ for all $v \in V$ and $\psi(v)^{T} R \psi(w)=0$ for all $v w \in E$. By Theorem 2.4, there exist vectors $y_{1}, y_{2}$ in the linear span of $z_{1}, z_{2}, z_{3}$ such that $\operatorname{supp}\left(y_{1}\right)$ and $\operatorname{supp}\left(y_{2}\right)$ are separated. This contradiction shows that all diagonal are nonzero.

If the diagonal entries are all 1 or all -1 , then $\phi(v)^{T} N \phi(v) \neq 0$ if $\phi(v) \neq 0$. Suppose three of the diagonal entries are 1 and one of them is -1 ; without loss of generality, we may assume that $n_{1,1}=-1$ and $n_{i, i}=1$ for $i=2,3,4$. Let $u w$ be an edge in $G$ such $\phi(u)$ and $\phi(w)$ are linearly independent. Let $a, b \in \mathbb{R}$ be such that $a \phi(u)+b \phi(w)$ is a vector in $\mathbb{R}^{n}$ whose first coordinate is equal to 0 . Since $\phi(u)^{T} N \phi(u)=0, \phi(w)^{T} N \phi(w)=0$, and $\phi(u)^{T} N \phi(w)=0, \quad(a \phi(u)+$ $b \phi(w))^{T} N(a \phi(u)+b \phi(w))=0$. However, since $n_{i, i}=1$ for $i=2,3,4$ and the first coordinate of $a \phi(u)+b \phi(w)$ equals $0,(a \phi(w)+b \phi(w))^{T} N(a \phi(u)+b \phi(w)) \neq 0$; a contradiction. The case where three of the diagonal entries are -1 and one of them is 1 is similar. Thus, two of the diagonal entries are -1 and two of the diagonal entries are 1; we may assume that $n_{1,1}=n_{2,2}=1$ and $n_{3,3}=n_{4,4}=-1$. Let

$$
A=\left[\begin{array}{cccc}
1 & 0 & 0 & \frac{1}{2} \\
0 & 1 & \frac{1}{2} & 0 \\
0 & -1 & \frac{1}{2} & 0 \\
-1 & 0 & 0 & \frac{1}{2}
\end{array}\right]
$$

then $A^{T} N A=Q$. Defining $\psi: V \rightarrow \mathbb{R}^{4}$ by $\psi(i)=A^{-1} \phi(i)$ for $i=1,2, \ldots, n$, we obtain that $\psi(i)^{T} Q \psi(i)=0$ for all $i \in V$ and $\psi(i)^{T} Q \psi(j)=0$ for all $i j \in E$. 
3. The parameter $\mu(G)$ and the Strong Arnol'd Hypothesis. In this section we apply Theorems 2.3 and 2.4 to show that if $G$ is a path, 2-connected outerplanar, or 3-connected planar, then each matrix in $\mathcal{O}(G)$ with exactly one negative eigenvalue satisfies the Strong Arnol'd Hypothesis. Different proofs can be found in [8].

For $x \in \mathbb{R}^{n}$, we denote $\operatorname{supp}_{-}(x)=\left\{i \mid x_{i}<0\right\}$ and $\operatorname{supp}_{+}(x)=\left\{i \mid x_{i}>0\right\}$. If $G=(V, E)$ is a connected graph with $V=\{1,2, \ldots, n\}$, then the Perron-Frobenius Theorem says that each eigenvector $z$ belonging to the smallest eigenvalue of $M \in$ $\mathcal{O}(G)$ has multiplicity 1 and satisfies $z>0$ or $z<0$. Since any $x \in \operatorname{ker}(M)$ is orthogonal to $z, \operatorname{supp}_{+}(x) \neq \emptyset$ and $\operatorname{supp}_{-}(x) \neq \emptyset$ for every nonzero $x \in \operatorname{ker}(M)$.

Lemma 3.1. [9, Theorem $2.17(\mathrm{v})]$ Let $G$ be a connected graph and let $M \in \mathcal{O}(G)$ with exactly one negative eigenvalue. Let $x \in \operatorname{ker}(M)$ be such that $G\left[\operatorname{supp}_{+}(x)\right]$ or $G\left[\operatorname{supp}_{-}(x)\right]$ has at least two components. Then there is no edge connecting $\operatorname{supp}_{+}(x)$ and $\operatorname{supp}_{-}(x)$ and $N(K)=N(\operatorname{supp}(x))$ for each component $K$ of $G[\operatorname{supp}(x)]$.

Lemma 3.2. Let $G=(V, E)$ be a graph and let $M \in \mathcal{O}(G)$ with exactly one negative eigenvalue. If $M$ has nullity at most three and there exists a nonzero $x \in$ $\operatorname{ker}(M)$ such that $\operatorname{supp}(x)$ induces a connected subgraph of $G$, then $M$ satisfies the Strong Arnol'd Hypothesis.

Proof. For the sake of contradiction, assume that there is an $M \in \mathcal{O}(G)$ that does not satisfy the Strong Arnol'd Hypothesis. By Lemma 2.7, there exists a nonzero vector $y \in \operatorname{ker}(M) \operatorname{such}$ that $\operatorname{supp}(x)$ and $\operatorname{supp}(y)$ are separated. The vector $z=x+y$ has the property that $G\left[\operatorname{supp}_{+}(z)\right]$ and $G\left[\operatorname{supp}_{-}(z)\right]$ are disconnected. By Lemma 3.1, $N(C)=N(\operatorname{supp}(z))$ for each component $C$ in $G\left[\operatorname{supp}_{-}(z)\right] \cup G\left[\operatorname{supp}_{+}(z)\right]$ and there is no edge between $\operatorname{supp}_{-}(z)$ and $\operatorname{supp}_{+}(z)$. However, this would mean that $G\left[\operatorname{supp}_{-}(x)\right]$ and $G\left[\operatorname{supp}_{+}(x)\right]$ are separated, contradicting the connectedness of $G[\operatorname{supp}(x)]$. $\mathrm{c}$

For a graph $G=(V, E)$ and an $S \subseteq V$, we denote by $G-S$ the subgraph of $G$ induced by the vertices in $V \backslash S$.

Theorem 3.3. Let $G=(V, E)$ be a graph which has no vertex cut $S$ such that $G-S$ has at least four components, each of which is adjacent to every vertex in $S$. Then every $M \in \mathcal{O}(G)$ with nullity at most three and with exactly one negative eigenvalue satisfies the Strong Arnol'd Hypothesis.

Proof. For the sake of contradiction, assume that there is an $M \in \mathcal{O}(G)$ that does not satisfy the Strong Arnol'd Hypothesis.

By Lemma 3.2, $G[\operatorname{supp}(x)]$ is disconnected for each nonzero $x \in \operatorname{ker}(M)$. For every $x \in \operatorname{ker}(M)$, there are at most three components in $G[\operatorname{supp}(x)]$, by assumption and by Lemma 3.1. By Theorem 2.6, for every nonzero vectors $x, y \in \operatorname{ker}(M)$, any 
component $C$ of $G[\operatorname{supp}(x)]$ and any component $D$ of $G[\operatorname{supp}(y)]$, either $C=D$, or $C$ and $D$ are separated, for otherwise $M$ would satisfy the Strong Arnol'd Hypothesis. Hence, we can conclude that there are at most three mutually disjoint connected subgraphs $K_{1}, K_{2}, K_{3}$ of $G$ such that for every $x \in \operatorname{ker}(M), G\left[\operatorname{supp}_{+}(x)\right]$ can be written as the union of some of $K_{1}, K_{2}, K_{3}$. We now show that $\operatorname{ker}(M)$ has dimension at most two.

For any $x \in \operatorname{ker}(M)$ and any $K_{i}, M_{K_{i}} x_{K_{i}}=0$, and hence, by the PerronFrobenius Theorem, $x_{K_{i}}<0, x_{K_{i}}=0$, or $x_{K_{i}}>0$. Furthermore, the eigenvalue 0 has multiplicity 1 in $M_{K_{i}}$. Let $z$ be a positive eigenvector belonging to the negative eigenvalue of $M$. Since $x^{T} z$ for any $x \in \operatorname{ker}(M), \operatorname{ker}(M)$ has dimension at most two. If $M$ does not satisfy the Strong Arnol'd Hypothesis, then, by Theorem 2.3, there are two nonzero vectors $x, y \in \operatorname{ker}(M)$ such that $G[\operatorname{supp}(x)]$ and $G[\operatorname{supp}(y)]$ are separated. Let $w=x+y$. Since $G[\operatorname{supp}(x)]$ and $G[\operatorname{supp}(y)]$ are disconnected, $G[\operatorname{supp}(w)]$ consists of at least four components. This contradicts the assumption in the theorem.

For a matrix $M$, we denote by nullity $(M)$ the nullity of $M$.

Corollary 3.4. Let $G=(V, E)$ be a graph and let $M \in \mathcal{O}(G)$ have $k:=$ $\operatorname{nullity}(M) \leq 3$. If $G$ has no $K_{4, k}$-minor, then $M$ satisfies the Strong Arnol'd Hypothesis.

We use this corollary to show that if $G$ is a path, 2-connected outerplanar, or 3 -connected planar, then each matrix in $\mathcal{O}(G)$ with exactly one negative eigenvalue satisfies the Strong Arnol'd Hypothesis.

TheOREM 3.5. [6] If $G$ is a path, then each $M \in \mathcal{O}(G)$ has nullity $(M) \leq 1$.

Since each 1-dimensional linear subspace $L \subseteq \mathbb{R}^{n}$ satisfies the Strong Arnol'd Hypothesis, we obtain:

Corollary 3.6. If $G$ is a path, then every matrix in $\mathcal{O}(G)$ satisfies the Strong Arnol'd Hypothesis.

A graph $G$ is outerplanar if it has an embedding in the plane such that each vertex is incident with the infinite face. Outerplanar graphs can be characterized as those graphs that have no $K_{4}$ - or $K_{2,3}$-minor.

Theorem 3.7. [7, Corollary 13.10.4] Let $G$ be a graph and let $M \in \mathcal{O}(G)$ with exactly one negative eigenvalue. If $G$ is 2 -connected outerplanar, then nullity $(M) \leq$ 2 .

Corollary 3.8. Let $G$ be a 2-connected outerplanar graph. Then every matrix in $\mathcal{O}(G)$ with exactly one negative eigenvalue satisfies the Strong Arnol'd Hypothesis.

Planar graphs can be characterized as those graphs that have no $K_{5^{-}}$or $K_{3,3^{-}}$ 
minor.

Theorem 3.9. [7, Corollary 13.10.2] Let $G$ be a graph and let $M \in \mathcal{O}(G)$ with exactly one negative eigenvalue. If $G$ is 3-connected planar, then nullity $(M) \leq 3$.

COROLlary 3.10. Let $G$ be a 3-connected planar graph. Then every matrix in $\mathcal{O}(G)$ with exactly one negative eigenvalue satisfies the Strong Arnol'd Hypothesis.

An embedding of a graph in 3-space is linkless if each pair of disjoint circuits has zero linking number under the embedding; see Robertson, Seymour, and Thomas [10]. In the same paper they characterized graphs that have a linkless embedding as those graphs that have no minor isomorphic to a graph in the Petersen family, a family of seven graphs, one of which is the Petersen graph. We conclude with a conjecture.

Conjecture 3.11. Let $G$ be a 4-connected graph that has a linkless embedding. Then every matrix in $\mathcal{O}(G)$ with exactly one negative eigenvalue satisfies the Strong Arnol'd Hypothesis.

\section{REFERENCES}

[1] F. Barioli, S. Fallat, and L. Hogben. A variant on the graph parameters of Colin de Verdière: implications to the minimum rank of graphs. Electron. J. Linear Algebra, 13:387-404, 2005.

[2] Y. Colin de Verdière. Sur un nouvel invariant des graphes et un critère de planarité. J. Comb. Theory, Ser. B., 50:11-21, 1990.

[3] Y. Colin de Verdière. On a new graph invariant and a criterion of planarity. In N. Robertson and P. Seymour, editors, Graph Structure Theory, Contemporary Mathematics, American Mathematical Society, Providence, 147:137-147, 1993.

[4] Y. Colin de Verdière. Multiplicities of eigenvalues and tree-width of graphs. J. Comb. Theory, Ser. B., 74(2):121-146, 1998.

[5] R. Diestel. Graph Theory, 2nd edition. Springer-Verlag, New York, 2000.

[6] M. Fiedler. A characterization of tridiagonal matrices. Linear Algebra Appl., 2:191-197, 1969.

[7] C. Godsil and G. Royle. Algebraic Graph Theory. Springer-Verlag, New York, 2001.

[8] H. van der Holst. Topological and Spectral Graph Characterizations. PhD thesis, University of Amsterdam, 1996.

[9] H. van der Holst, L. Lovász, and A. Schrijver. The Colin de Verdière graph parameter. Graph Theory and Combinatorial Biology, Bolyai Soc. Math. Stud., 7:29-85, 1999.

[10] N. Robertson, P. Seymour, and R. Thomas. Sachs' linkless embedding conjecture. J. Comb. Theory, Ser. B., 64(2):185-227, 1995. 